\title{
Novel prognostic scores based on serum ferritin/globulin ratio in patients with hepatocellular carcinoma
}

\author{
Wen Liu ${ }^{1 \#}$, Qunxi Chen ${ }^{2 \#}$, Minjie Mao ${ }^{1}$, Runkun Han ${ }^{1}$, Yijun Liu ${ }^{1}$, Xueping Wang ${ }^{1}$ \\ ${ }^{1}$ Department of Laboratory Medicine, State Key Laboratory of Oncology in South China, Collaborative Innovation Center for Cancer Medicine, \\ Sun Yat-sen University Cancer Center, Guangzhou, China; ${ }^{2}$ Department of Pathology, State Key Laboratory of Oncology in South China, \\ Collaborative Innovation Center for Cancer Medicine, Sun Yat-sen University Cancer Center, Guangzhou, China \\ Contributions: (I) Conception and design: X Wang; (II) Administrative support: W Liu, X Wang; (III) Provision of study materials or patients: M Mao, \\ Q Chen; (IV) Collection and assembly of data: Y Liu; (V) Data analysis and interpretation: R Han; (VI) Manuscript writing: Y Liu, X Wang; (VII) \\ Final approval of manuscript: All authors. \\ \#These authors contributed equally to this work. \\ Correspondence to: Xueping Wang. Department of Clinical Laboratory, Sun Yat-sen University Cancer Center, State Key Laboratory of Oncology in \\ South China, Collaborative Innovation Center for Cancer Medicine, 651 Dong Feng Road East, Guangzhou 510060, China. \\ Email: wangxueping1226@163.com.
}

Background: The level of ferritin has been shown to be associated with the prognosis of various tumors. In this study, we proposed prognostic indicator, named ferritin/globulin ratio (FGR), and to evaluate the important value in hepatocellular carcinoma (HCC).

Methods: A total of 472 HCC patients and 219 healthy controls were concluded in our retrospective study. The clinical characteristics were analyzed in these patients. The optimal cutoff value of the prognostic factors ( $\alpha$-fetoprotein, alanine aminotransferase, aspartate aminotransferase, ferritin, globulin and FGR) were determined by using receiver operating characteristic curve analysis, and then analyzed by univariate and multivariate Cox hazard models to evaluate the prognostic significance.

Results: Serum ferritin, globulin and FGR levels were significantly higher in HCC patients than in healthy controls. Multivariate analysis showed that FGR was a significant and independent risk factor OS $(\mathrm{HR}=1.575 ; 95 \% \mathrm{CI}: 1.122-2.212 ; \mathrm{P}=0.009)$ and DFS (HR =1.569; 95\% CI: 1.117-2.204; P=0.009) in whole patients. Furthermore, we also classified the data according to $\alpha$-fetoprotein (AFP), alanine aminotransferase (ALT) and aspartate aminotransferase (AST), highly significant differences of FGR were observed between the two groups in OS and DFS. High FGR levels were associated with poor OS and DFS in HCC patients.

Conclusions: The serum FGR levels may serve as a significant predictor of prognosis in HCC patients.

Keywords: Hepatocellular carcinoma (HCC); ferritin; globulin; prognosis; overall survival (OS)

Submitted Feb 11, 2020. Accepted for publication Aug 21, 2020.

doi: $10.21037 /$ tcr-20-966

View this article at: http://dx.doi.org/10.21037/tcr-20-966

\section{Introduction}

Hepatocellular carcinoma (HCC), which arises from the liver cells (hepatocytes) and accounts for around $80-90 \%$ of all liver cancers. The incidence of HCC is increasing by $3-9 \%$ year by year in global (1). HCC is one of the most malignant tumors and has the highest morbidity and mortality rates of any cancer worldwide, with the 5-year overall survival (OS) is less than $10 \%$ (2). Because the earlystage disease is usually asymptomatic, and the symptoms of early-stage disease are nonspecific, approximate half of patients are diagnosed at an advanced stage. Take the poor prognosis of HCC into account, effective biomarkers needed to be identified to guide individualized treatment of HCC patients and then improve their prognosis.

Many biomarkers, such as $\alpha$-fetoprotein (AFP) and 
liver function, such as alanine aminotransferase (ALT) and aspartate aminotransferase (AST), have already been used to screen for HCC and choose the best treatment on an individual status. However, these biomarkers are not sufficient to predict prognosis accurately $(3,4)$. Serum ferritin (FERR) is the oldest known protein involved in iron metabolism, and a multi-functional protein with possible roles in proliferation, angiogenesis, immunosuppression, and iron delivery. The abnormal FERR level has been shown to be closely correlated with tumor progression and prognosis in several cancers.

Kew MC declared that high level FERR is a potential biomarker for primary liver cancer (5), Weinstein RE reported that increased FERR contributed to the progression of breast cancer (6). The relationship between cancer and FERR is unclear. Furthermore, the iron accumulation is considered to play an important role in liver injury and hepatocarcinogenesis (7). Because the serum FERR is widely recognized as an acute phase reactant and marker of acute and chronic inflammation, and increased in the environment of inflammation (8), the level of FERR in HCC is influenced by tumor and inflammation. Owing to increasing evidence regarding the role of FERR in HCC, systemic biomarkers needed to be found to eliminate the effect of non-specific inflammation. Globulin, a predictor of inflammatory status, has been shown to be reflected poor OS of many cancers (9). Therefore, we have combined the FERR and globulin to provide a potent predictive system for the HCC prognosis after treatment.

In this study, the level of ferritin/globulin ratio (FGR) in HCC patients has been studied. To describe the association between FGR and HCC, we measured the FGR level in the pre-therapy serum of patients with HCC in comparison with those of healthy participants and performed a largescale retrospective cohort analysis. The flowchart of our study is showed in Figure S1. We present the following article in accordance with the REMARK reporting checklist (available at http://dx.doi.org/10.21037/tcr-20-966).

\section{Methods}

\section{Patients}

We retrospectively reviewed the clinical data of 472 HCC patients (420 men and 52 women; ages 19-88 years), who received histologically confirmed diagnosed at Sun Yat-Sen University Cancer Center from January 2009 and December 2012. As the Supplementary material shown the patients' selection process and describe the figure. The main clinical characteristics are described in Table 1. All of the patients met the diagnostic criteria for HCC. Exclusion criterion were as follows: (I) patients treated with surgery or received any treatment drugs or feeding before serum collection; (II) patients with concomitant diseases associated with serum ferritin and globulin levels (i.e., inflammation, gammopathy, MGUS or metabolic syndrome); (III) other types of malignancy. HCC staging was performed by the American Joint Committee on Cancer Staging system (AJCC, 2002; Greene, American Joint Committee on Cancer. American Cancer Society, 2002). All patients received treatment, including surgery, transarterial embolization (TAE), ablation therapy. Patient characteristics, preoperative serum AFP levels, ferritin levels, globulin levels, FGR, postoperative OS and disease-free survival (DFS) survival were recorded. Alcohol index was assessed as drinking or not drinking. Prior to use of these serum, informed consent was obtained from each of the patients. A total of 300 healthy participants (251 men and 49 women; ages 23-79 years, median 54 years) free of tumor and nutritional diseases (i.e., inflammation, metabolic syndrome or feeding) were recruited from the physical examination department at Sun Yat-Sen University Cancer Center. The study was conducted in accordance with the Declaration of Helsinki (as revised in 2013). This study was approved by the Institute Research Ethics Committee of the Sun Yat-Sen University Cancer Center, Guangzhou, China (approval No. 2017-FXY-129).

\section{Follow-up}

The primary outcome of our study was OS, defined as the time between the first diagnosis of HCC and death, or date of the last follow-up. Relatively, the secondary outcome was DFS, calculated from the date of first diagnosis to the date of disease recurrence. After completion of primary treatment, patients were generally followed up every 3 months in the first 2 years, every 6 months for the following 3 to 5 years, and annually thereafter for patients without evidence of recurrence. The last follow-up was in December 2016. The survival status was verified through checking clinical attendance records or direct telecommunication with the patient or their family (performed by The Medical Information Unit in our Cancer Center).

\section{Laboratory measurements}

As part of the physical examination, peripheral blood was 
Table 1 Characteristics and parameters of the HCC patients

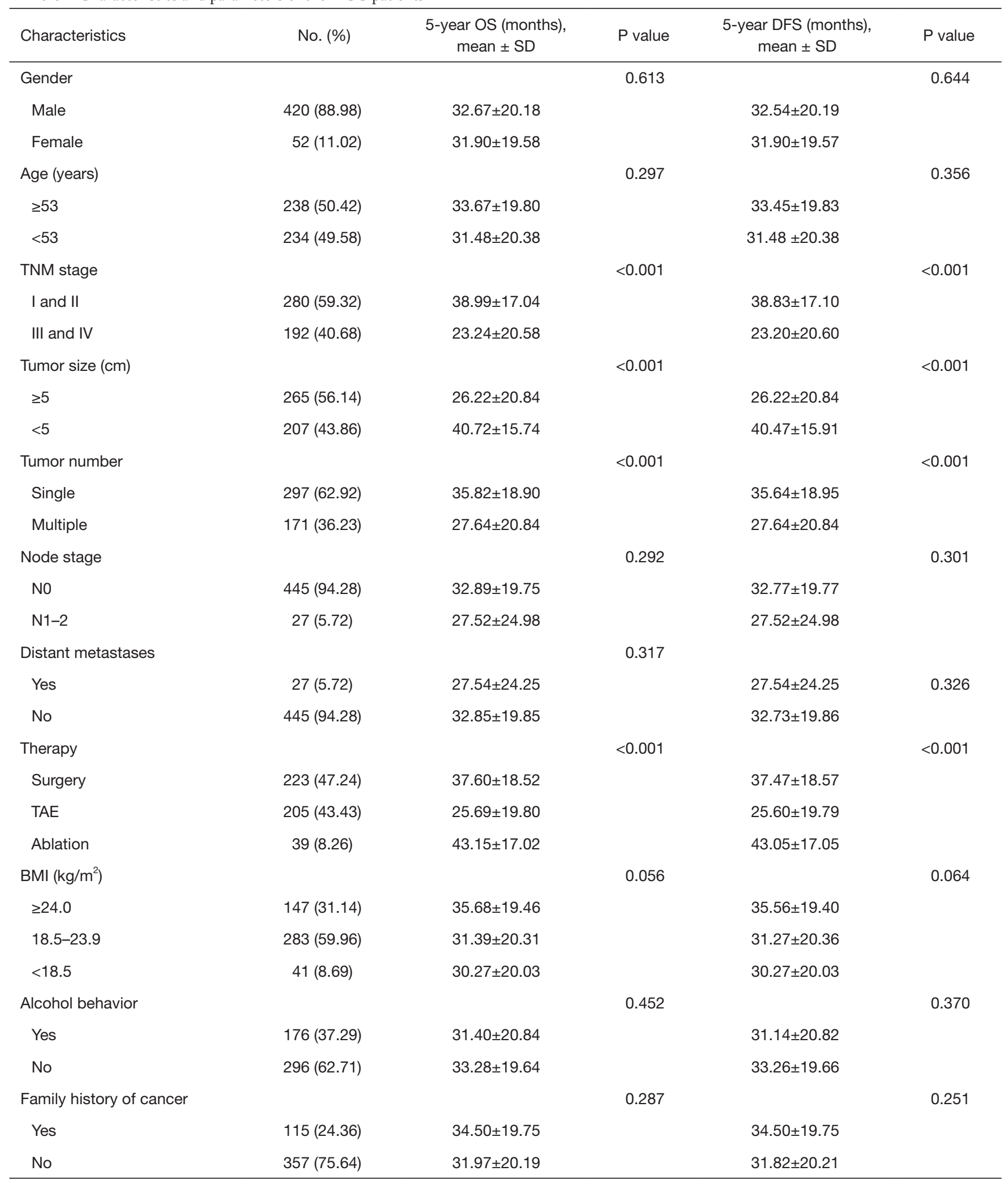

Table 1 (continued) 
Table 1 (continued)

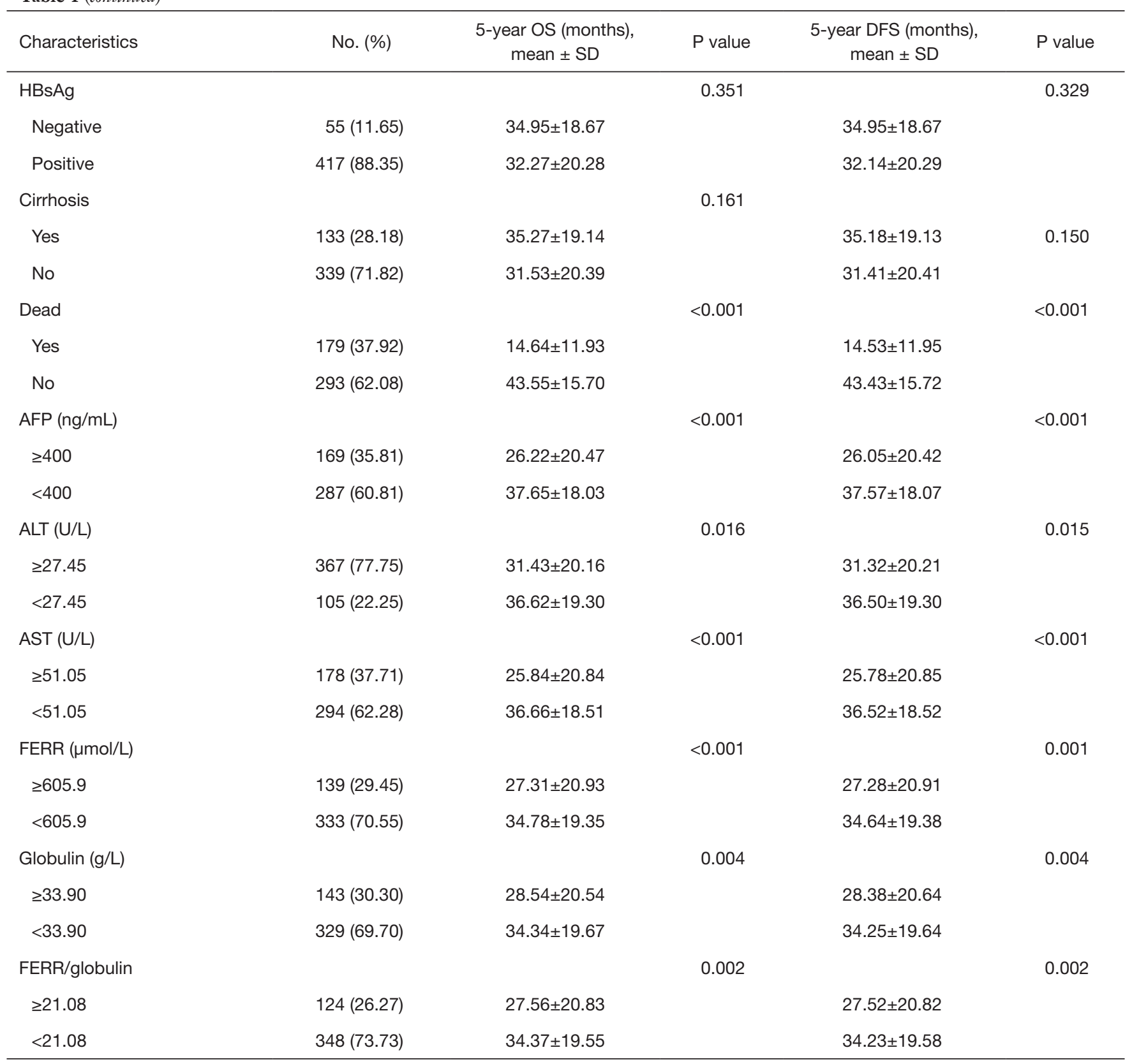

mean $\pm \mathrm{SD}$, mean \pm standard deviation. OS, overall survival; DFS, disease-free survival.

collected from the patients between 7 and 8 a.m., before treatment, clotted at room temperature, and centrifuged at 3,500 r/min for $8 \mathrm{~min}$. The levels of AFP and FERR were tested using ROCHE Modular Analytics E170 automatic electrochemistry analyzer (Basel, Switzerland). The level of ALT, AST and globulin were measured using a Hitachi 7600 automatic biochemical analyzer (Tokyo, Japan).

\section{Statistical analysis}

All statistical tests were performed with SPSS 16.0 for Windows software (SPSS, Chicago, IL, USA). Data were expressed as the mean and standard deviation (mean \pm SD). The cut-off value of the lipid AFP, FERR, globulin, ALT, AST, FGR were categorized by receiver operating characteristic (ROC) curve statistical analyses. The 

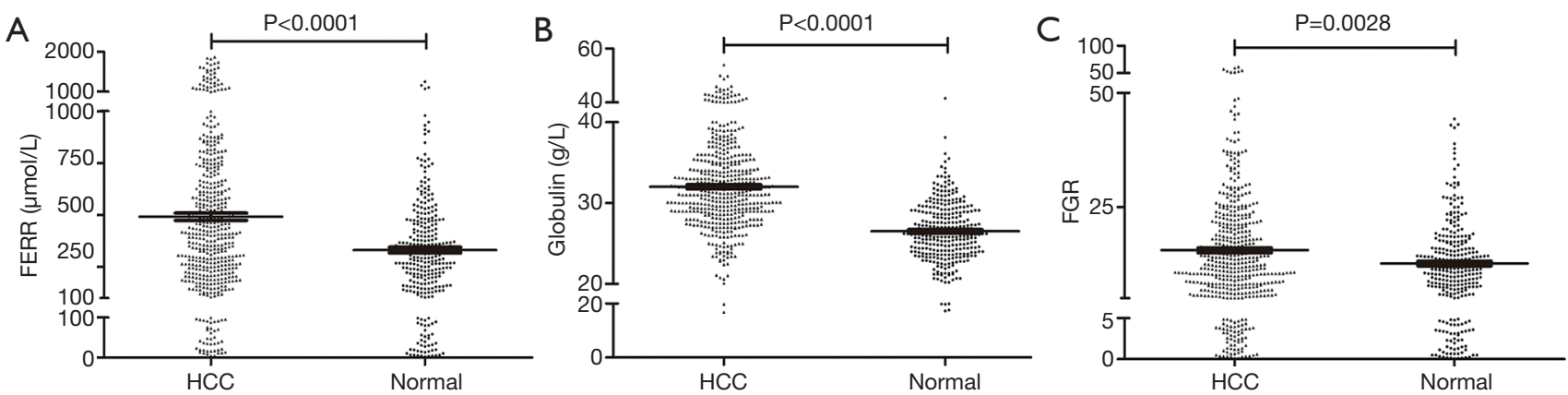

Figure 1 Pre-treatment serum FGR level in HCC patients and healthy controls. The serum FERR, globulin and FGR levels in HCC patients and healthy controls are plotted as a distribution (A,B,C). FGR, ferritin/globulin ratio; HCC, hepatocellular carcinoma; FERR, ferritin.

correlation between OS, DFS and clinical characteristics was assessed using the Mann-Whitney $U$ test. The differences between HCC patients and healthy donors were compared by the nonparametric test using Mann-Whitney $\mathrm{U}$ test. Univariate and multivariate analyses of clinical variables were performed using Cox proportional hazards regression models. The results of this survey were analyzed using the Kaplan-Meier survival curves with the logrank test and proportional hazard model. The correlation between FGR and clinical characteristics was analyzed using the Mann-Whitney $\mathrm{U}$ test and $\chi^{2}$ test. $\mathrm{P}$ values were derived from two-sided tests and $\mathrm{P}$ values $<0.05$ were regarded as statistically significant.

\section{Results}

\section{The basic clinical characteristics in HCC patients and subgroup}

The relationships between OS, DFS and clinical characteristics of the patients are summarized in Table 1. From January 2009 and December 2012, a total 472 patients with HCC cancer were enrolled in the analysis. The median age of the patients was 53 years (range, 1988 years), and $88.98 \%$ of patients were males. The numbers of patients at stage I-II and III-IV were $282(59.32 \%)$ and 192 (40.68\%), respectively. Moreover, the OS and DFS were significantly correlated with TNM stage $(\mathrm{P}<0.001$ vs. $\mathrm{P}<0.001)$, tumor size $(\mathrm{P}<0.001$ vs. $\mathrm{P}<0.001)$, tumor number $(\mathrm{P}<0.001$ vs. $\mathrm{P}<0.001)$, AFP $(\mathrm{P}<0.001$ vs. $\mathrm{P}<0.001)$, ALT $(\mathrm{P}=0.016$ vs. $\mathrm{P}=0.015)$, AST $(\mathrm{P}<0.001$ vs. $\mathrm{P}<0.001)$, FERR $(\mathrm{P}<0.001$ vs. $\mathrm{P}=0.001)$, globulin $(\mathrm{P}=0.004$ vs. $\mathrm{P}=0.004)$ and FGR ( $\mathrm{P}=0.002$ vs. $\mathrm{P}=0.002)$. There were 179 patients died and 6 patients recurrence; 223 patients received surgery,
205 patients received TAE, while 39 patients had ablation.

\section{Comparison of pre-treatment FGR between HCC patients and healthy controls}

The levels of pre-treatment FERR and globulin were compared between HCC patients and healthy controls using the nonparametric test, to investigate whether FGR abnormalities occur in HCC. The serum levels of FERR $(491.80 \pm 365.40 \mu \mathrm{mol} / \mathrm{L})$, globulin $(32.00 \pm 5.23 \mathrm{~g} / \mathrm{L}), \mathrm{FGR}$ $(15.53 \pm 11.29)$ in HCC patients were significantly higher than those in control group with age and sex matched (FERR: $331.60 \pm 225.50 \mu \mathrm{mol} / \mathrm{L}$, globulin: $26.50 \pm 3.60 \mathrm{~g} / \mathrm{L}$, FGR: $12.63 \pm 8.65$ ) (Figure 1).

\section{Associations between FGR and HCC patient survival analysis}

The potentially important factors identified in univariate analysis were included in the multivariate analysis (Cox proportional hazards model). The multivariate analysis showed that tumor size (HR $=2.029$; 95\% CI: $1.522-3.205$; $\mathrm{P}<0.001)$, tumor number (HR $=1.455$; 95\% CI: 1.045-2.026; $\mathrm{P}=0.026)$, AFP (HR =1.764; 95\% CI: 1.288-2.416; $\mathrm{P}<0.001$ ), therapy $(\mathrm{HR}=1.437 ; 95 \% \mathrm{CI}: 1.202-1.717 ; \mathrm{P}=0.026)$, BMI (HR $=0.746 ; 95 \%$ CI: 0.581-0.957; $\mathrm{P}=0.021)$, FGR (HR $=1.680 ; 95 \%$ CI: 1.214-2.325; $\mathrm{P}=0.002)$ was identified as significantly independent predictors of OS of HCC patients (Table 2) and also tumor size (HR $=2.012 ; 95 \%$ CI: $1.370-2.955 ; \mathrm{P}<0.001)$, tumor number $(\mathrm{HR}=1.636$; 95\% CI: 1.177-2.276; $\mathrm{P}=0.003)$, AFP $(\mathrm{HR}=1.831 ; 95 \%$ CI: $1.331-2.519 ; \mathrm{P}<0.001)$, AST $(\mathrm{HR}=1.510 ; 95 \% \mathrm{CI}$ : $1.048-2.177 ; \mathrm{P}=0.027), \mathrm{FGR}(\mathrm{HR}=1.569 ; 95 \% \mathrm{CI}$ : 1.117-2.204; $\mathrm{P}=0.009)$ were strongly associated with DFS 
Table 2 Univariate and multivariate cox hazards analysis for overall survival in 472 patients with HCC

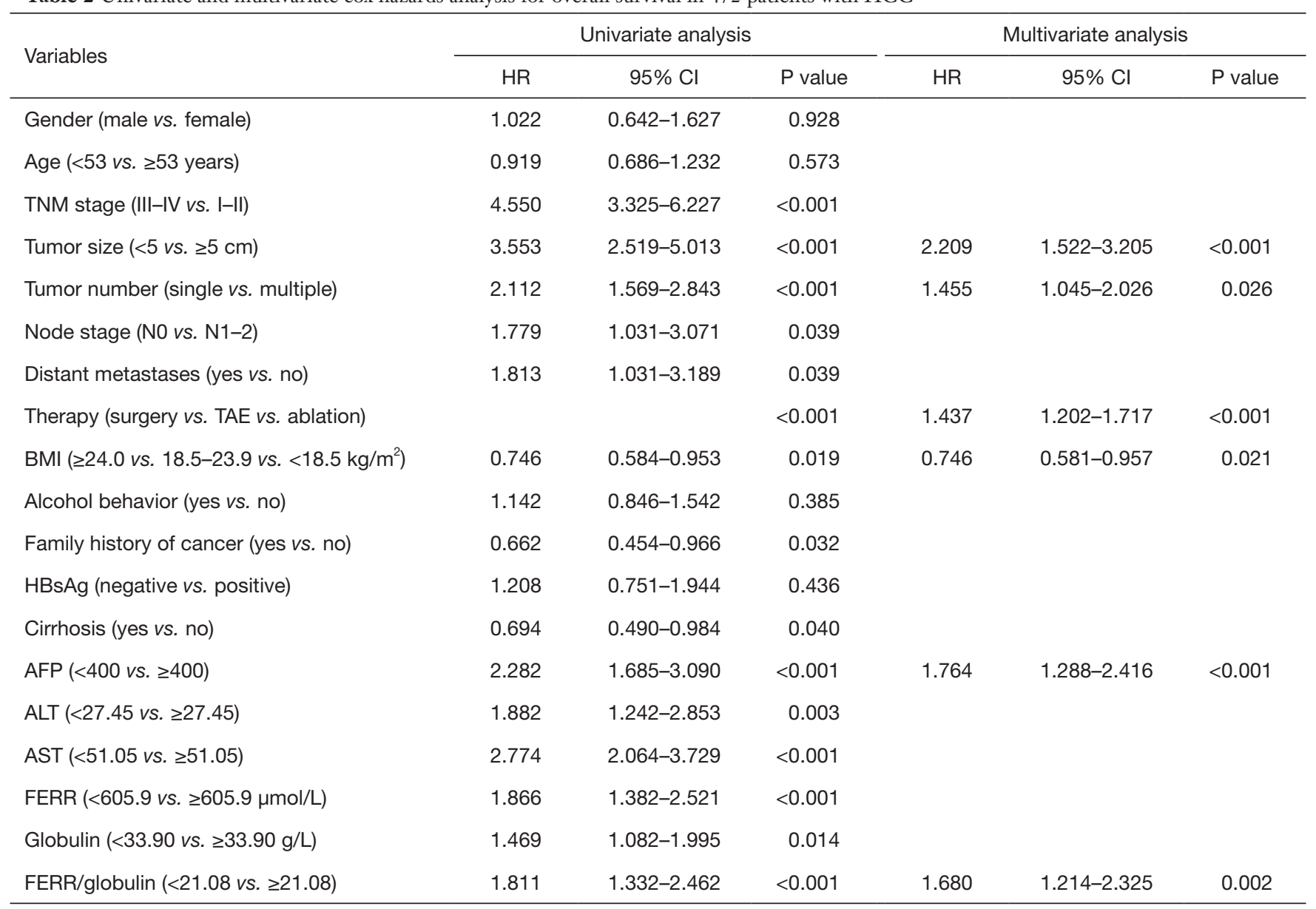

HCC, hepatocellular carcinoma.

(Table 3). Which were showed that FGR could be served as an independent prognostic factor in HCC patients.

To further explore the prognostic significance of FGR level in HCC, the Kaplan-Meier method was performed to plot the survival curves, and the groups were compared using the log rank test. In the whole cohort, the mean OS of the low FRG group was 34.37 months while the high FGR group was 27.56 months $(\mathrm{P}=0.002)$, and the mean DFS of the low FGR was 34.23 months while the high FGR group was 27.52 months $(\mathrm{P}=0.002)$. The results show that patients with decreased FGR levels were significantly associated with better OS $(\mathrm{P}<0.001)$ and DFS $(\mathrm{P}<0.001)$ than the increased FGR group. Patients with tumor size $<5 \mathrm{~cm}$ (OS: $\mathrm{P}<0.001$ vs. DFS: $\mathrm{P}<0.001$ ), single tumor (OS: $\mathrm{P}<0.001$ vs. DFS: $\mathrm{P}<0.001$ ), lower AFP levels (OS: $\mathrm{P}<0.001$ vs. DFS: $\mathrm{P}<0.001$ ) and lower AST (OS: $\mathrm{P}<0.001$ vs. DFS: $\mathrm{P}<0.001$ ) had better prognosis, Figures 2,3.

\section{The relationship between the FGR and clinicopathologic characteristics in HCC patients}

The associations between plasma FGR and clinicopathological variables in 472 HCC patients are further analyzed in Table 4. The FGR levels were higher in males, advanced stage $(\mathrm{P}<0.001)$, large tumor size $(\mathrm{P}<0.001)$, Node metastasis ( $\mathrm{P}=0.003), \mathrm{HBV}$ Ag positive $(\mathrm{P}=0.037)$, high ALT $(\mathrm{P}=0.006)$, high AST $(\mathrm{P}<0.001)$, and not associated with age, tumor number, distant metastases, alcohol behavior, BMI, family history of cancer, cirrhosis and AFP. Furthermore, all the patients were divided into two groups by the FGR cut-off (21.08). Gender, age, tumor number, distant metastases, alcohol behavior, BMI, family history of cancer, cirrhosis and AFP were similar between the two groups. However, patients in advanced stage with an increased FGR than early stage of HCC $(\mathrm{P}<0.001)$, and patients with HBV Ag 
Table 3 Univariate and multivariate cox hazards analysis for Disease-free survival in 472 patients with HCC

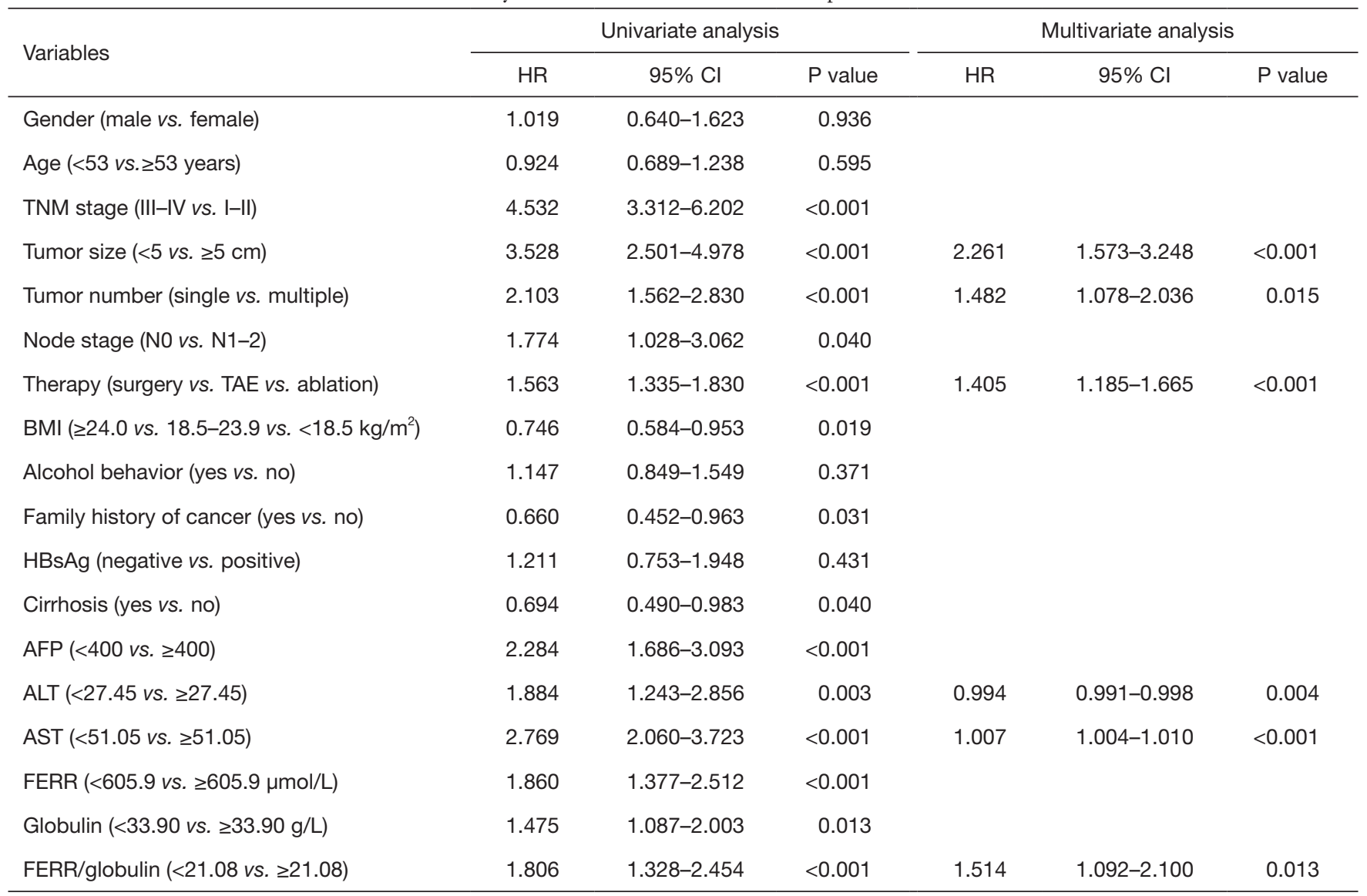

HCC, hepatocellular carcinoma; HR, hazard ratio; 95\% Cl, 95\% confidence interval.

positive, higher ALT and higher AST also have a higher FGR than HBV Ag negative, low ALT and low AST ( $\mathrm{P}=0.037, \mathrm{P}=0.031, \mathrm{P}<0.001$, respectively).

\section{Prognostic significance of FGR according to pathological stage, ALT, and AST}

To explore the subgroups of patients according to pathological stage, ALT, and AST, whether were influenced by FGR levels. Patients with decreased FGR $(<21.08)$ showed a better OS $(\mathrm{P}=0.045)$ and DFS $(\mathrm{P}=0.046)$ compared with patients with higher FGR level for advanced pathological stage (stage III and IV) (Figure 4). Both OS and DFS were poor when patients had high ALT level $(\geq 27.45 \mathrm{U} / \mathrm{L})$ and high FGR $(\mathrm{P}=0.001$ and $\mathrm{P}=0.001)$ (Figure 5). When AST $\geq 51.05 \mathrm{U} / \mathrm{L}$ and FGR $\geq 21.08$, HCC patients showed significantly poor OS and DFS $(\mathrm{P}=0.016$ and $\mathrm{P}=0.016$ ) (Figure 6).

\section{The prognostic value of the combining FGR and ALT/AST}

We classified the patients into the three subgroups: score 1 (low risk: low FGR and low ALT), score 2 (medium risk: low FGR or low ALT), score 3 (high risk: high FGR and high ALT). Patients with a lower FGR and ALT score had a significantly shorter OS compared with those patients with a high score. Moreover, we also classified the patients into the three subgroups: score 1 (low risk: low FGR and low AST), score 2 (medium risk: low FGR or low AST), score 3 (high risk: high FGR and high AST). Patients with a lower FGR and AST score had a significantly shorter OS compared with those patients with a high score (Figure 7).

\section{Discussion}

HCC is a heterogeneous solid tumor which is the main detoxifying organ in our body. Despite the improvement 

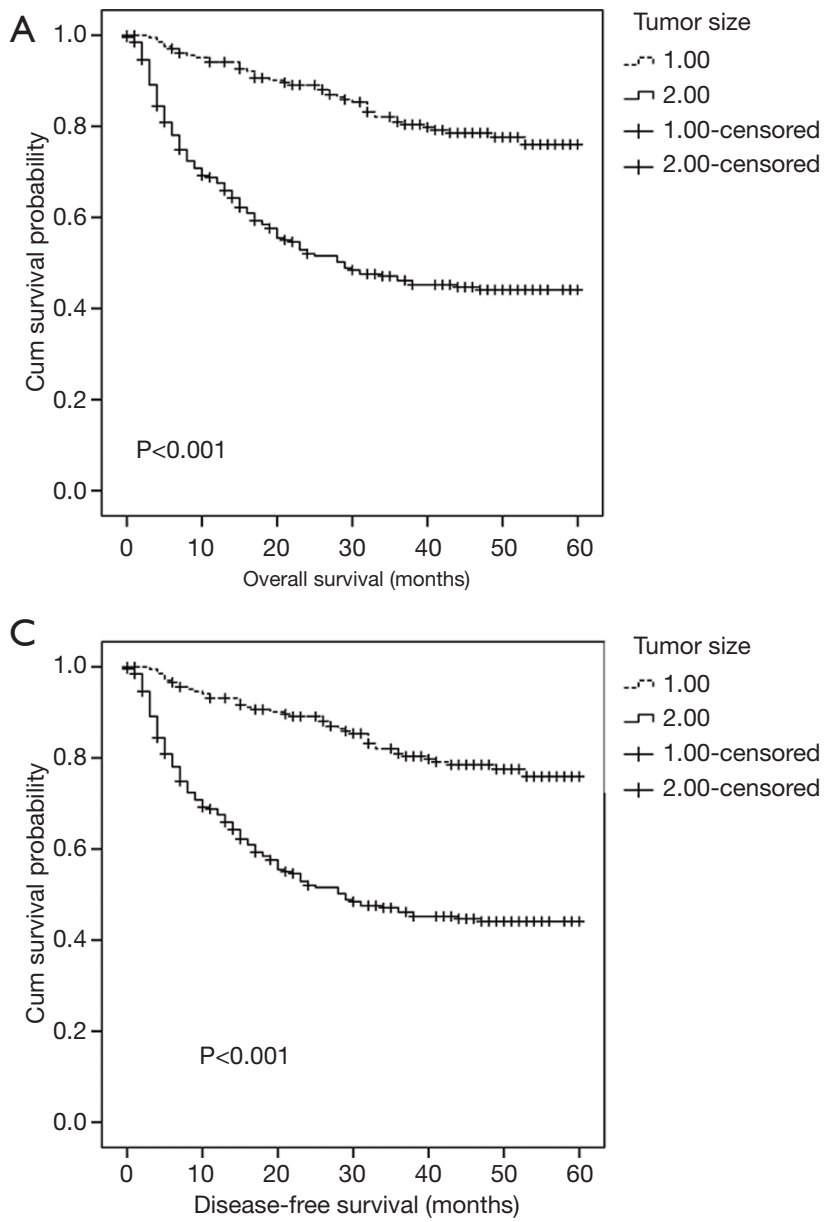

B

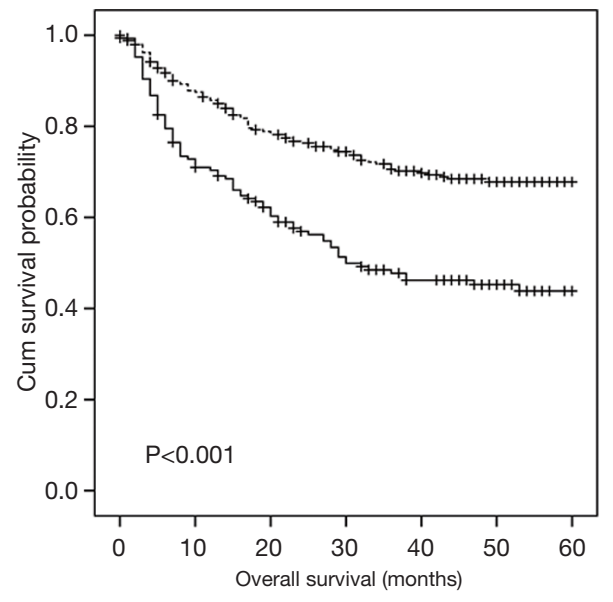

D

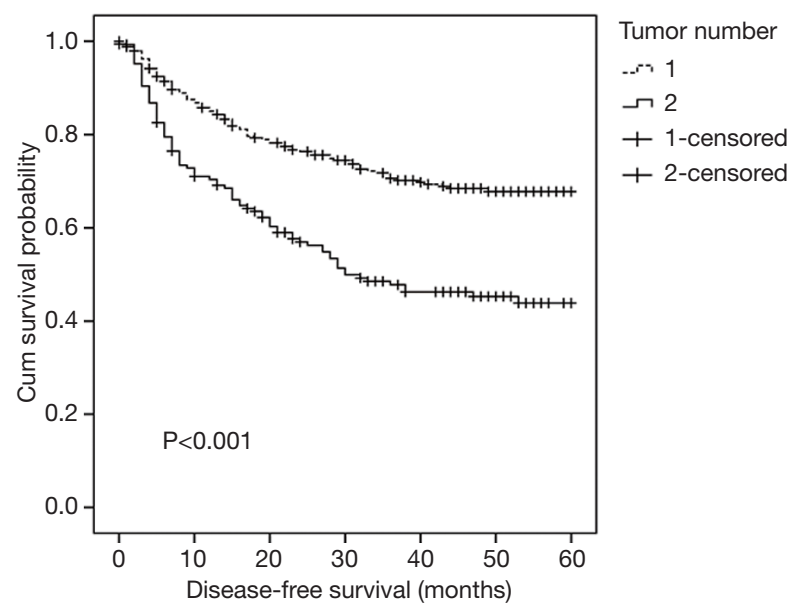

Figure 2 The OS and DFS of tumor size and tumor number in HCC patients by Kaplan-Meier method. (A) Analysis of OS in tumor size; (B) analysis of OS in tumor number; (C) analysis of DFS in tumor size; (D) analysis of DFS in tumor number. OS, overall survival; DFS, disease-free survival; HCC, hepatocellular carcinoma.

in diagnose and treatment strategy, the survival of patients with HCC suffering from hepatic carcinectomy remains poor (10). It is worth studying the risk of mortality and recurrence in order to reduce the rate of relapse and improve the quality of survival for patients with HCC $(11,12)$. FERR is played a key role in iron metabolism. Several studies, including HCC, have confirmed that abnormal ferritin levels associated with tumor occurrence, development and prognosis.

In this study, the results showed that FERR, globulin and FGR levels were significant higher in HCC patients than normal controls. Furthermore, OS and DFS were significantly poorer in patients with increased FGR levels than HCC patients with low FGR levels, thus FGR was the independent prognosis marker in HCC patients. In addition, we found that patients with large tumor size, multiple tumor number, increased AFP and AST levels experienced significantly shorter OS and DFS. Also, we analyzed the correlation between FGR and clinical characteristics, the present data showed that the FGR level is significantly associated with sex, pathological stage, HBV Ag infection, ALT and AST levels. Then, we analyzed the prognostic significance of FGR according to pathological stage, ALT, and AST. We found that Patients with decreased FGR showed a better OS and DFS compared with patients with higher FGR level for advanced pathological stage (stage III and IV). Both OS and DFS were better when patients had low ALT level and low FGR. When AST $<51.05$ U/L and FGR <21.08, HCC patients showed significantly high OS and DFS.

The present data show that FGR level are strongly associated with OS and DFS in HCC patients, and both FERR and globulin are convenient inexpensive indicators 
A

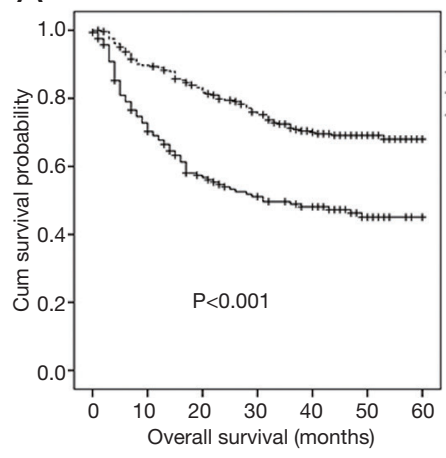

D

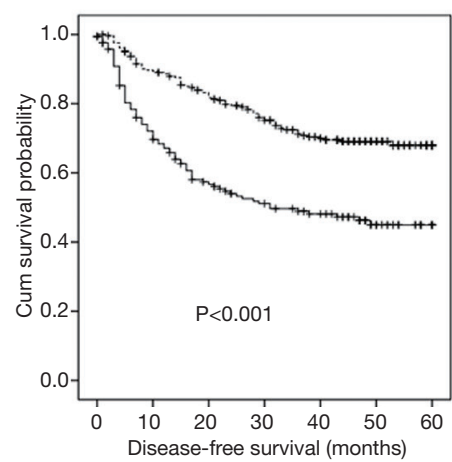

B

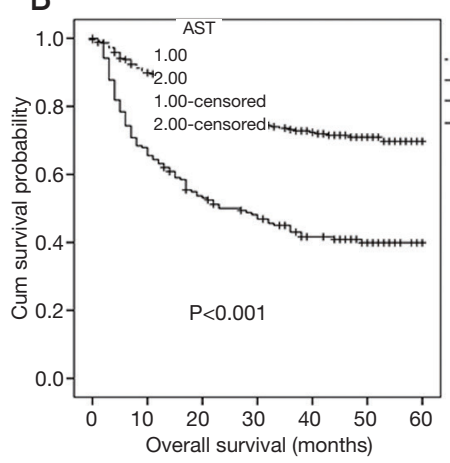

E
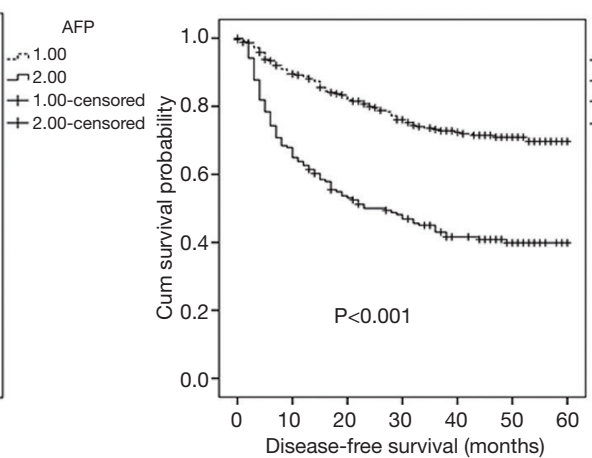

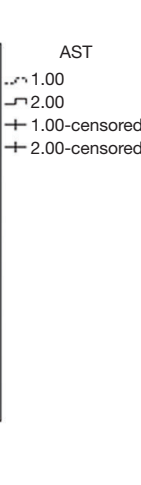

C

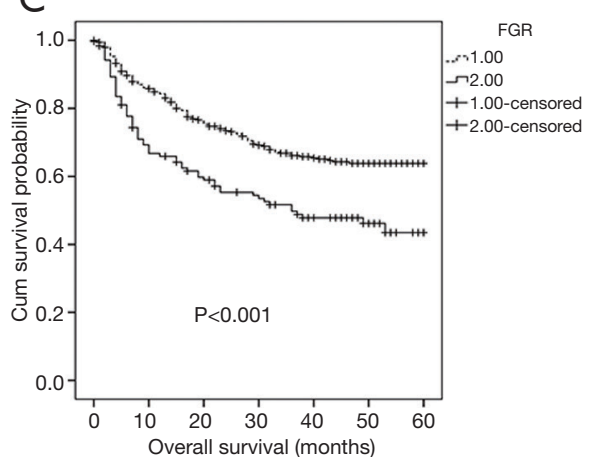

$\mathrm{F}$

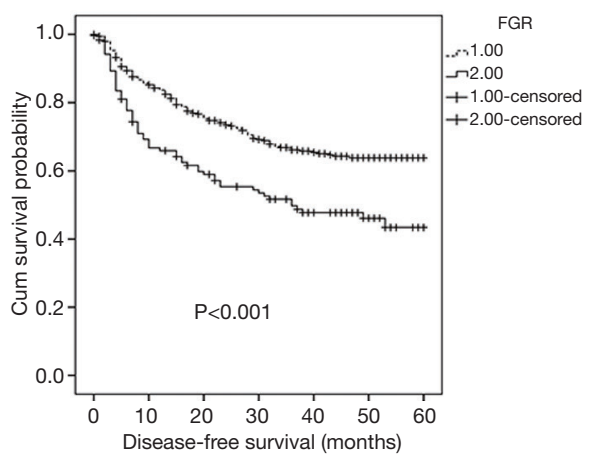

Figure 3 The OS and DFS of AFP, AST and FGR in HCC patients by Kaplan-Meier method. (A) Analysis of OS in AFP; (B) analysis of OS in AST; (C) analysis of OS in FGR; (D) analysis of DFS in AFP; (E) analysis of DFS in AST; (F) analysis of DFS in FGR. OS, overall survival; DFS, disease-free survival; AFP, $\alpha$-fetoprotein; AST, aspartate aminotransferase; FGR, ferritin/globulin ratio; HCC, hepatocellular carcinoma.

Table 4 Relationship between the FGR levels and the clinical characteristics in patients with HCC

\begin{tabular}{|c|c|c|c|c|c|c|}
\hline \multirow{2}{*}{ Characteristics } & \multicolumn{6}{|c|}{ FERR/Globulin } \\
\hline & Cases (n) & $<21.08$ & $\geq 21.08$ & $P$ value* & Mean \pm SD & $P$ value ${ }^{\star \star}$ \\
\hline Gender(n) & & & & 0.059 & & $<0.001$ \\
\hline Male & 420 & 304 & 116 & & $16.19 \pm 11.39$ & \\
\hline Female & 52 & 44 & 8 & & $9.80 \pm 8.81$ & \\
\hline$\geq 53$ & 238 & 176 & 62 & & $14.87 \pm 11.22$ & \\
\hline$<53$ & 234 & 172 & 62 & & $16.22 \pm 11.40$ & \\
\hline Stage (n) & & & & $<0.001$ & & $<0.001$ \\
\hline I and II & 280 & 226 & 54 & & $13.85 \pm 10.67$ & \\
\hline$\geq 5$ & 265 & 186 & 79 & & $16.59 \pm 11.65$ & \\
\hline$<5$ & 207 & 162 & 45 & & $14.28 \pm 10.80$ & \\
\hline
\end{tabular}

Table 4 (continued) 
Table 4 (continued)

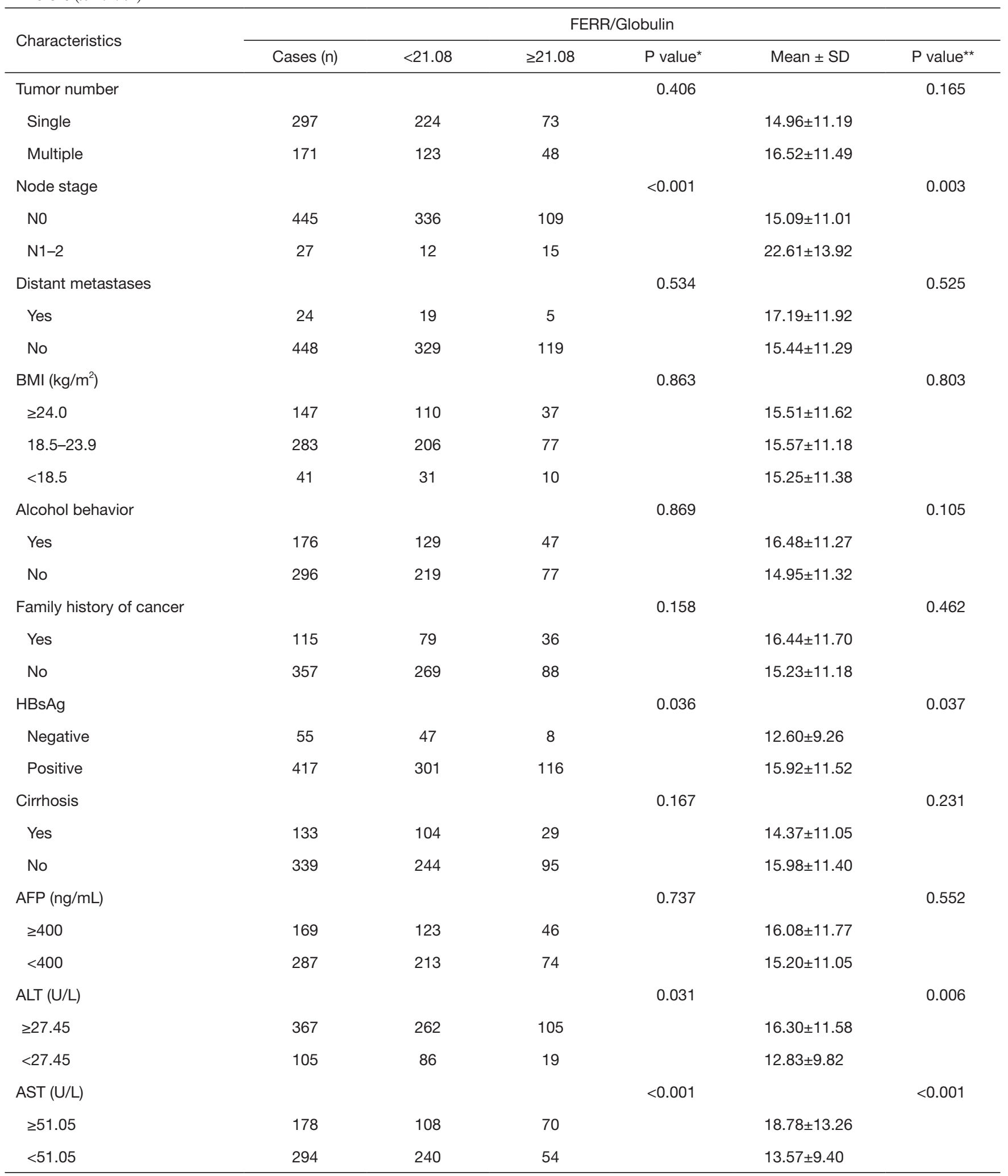

Mean $\pm \mathrm{SD}$, mean \pm standard deviation. ${ }^{*}, \mathrm{P}$ values were calculated using the chi-squared test $\left(\chi^{2}\right.$ test), $\mathrm{P}<0.05$ indicated significant differences. ${ }^{* *}, \mathrm{P}$ values were calculated using unpaired Student's $t$-tests or Mann-Whitney $\mathrm{U}$ test, $\mathrm{P}<0.05$ indicated significant differences. 

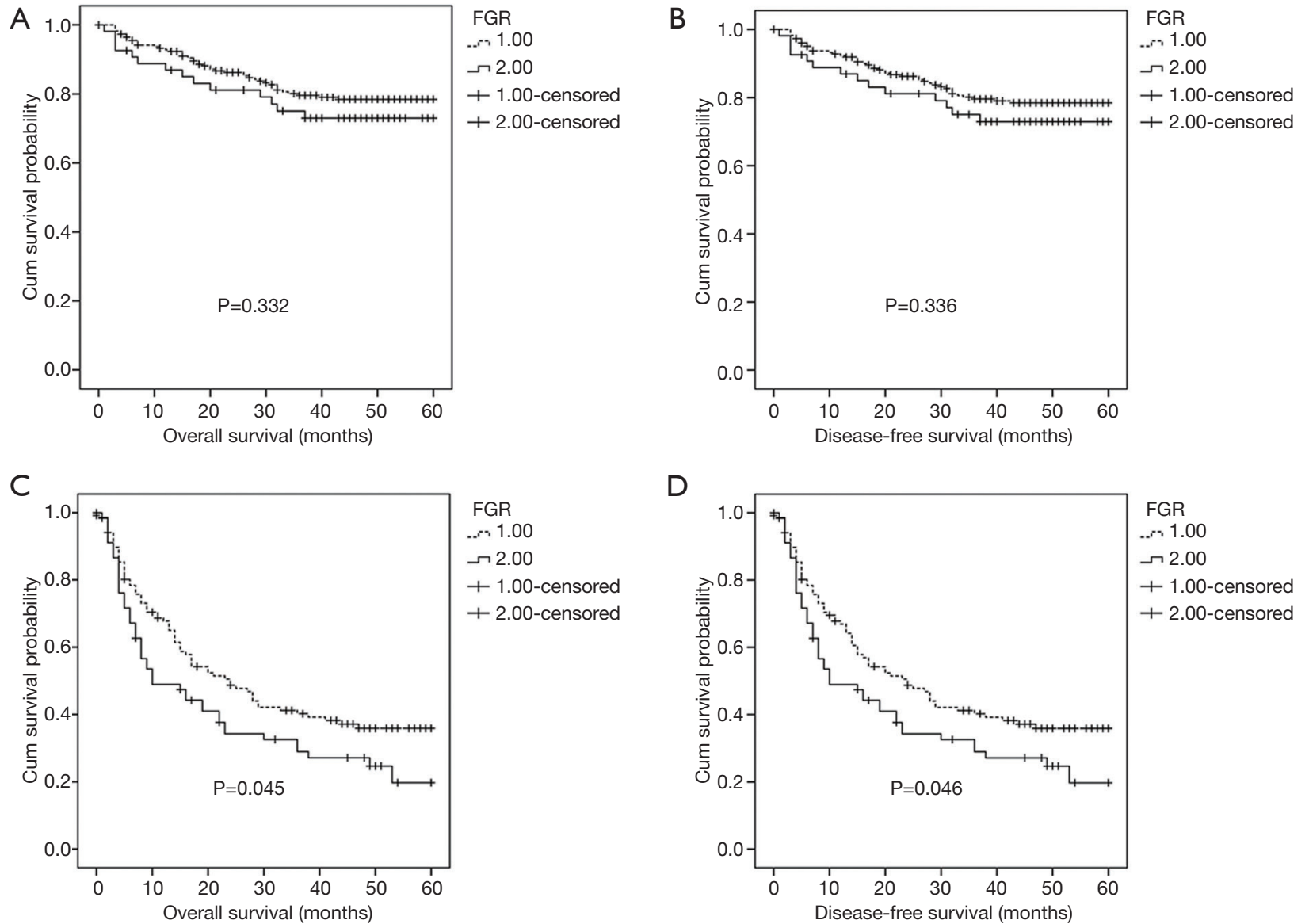

Figure 4 Prognosis significance of FGR level in different pathological stage. (A) OS of FGR level in early stage (stage I and II); (B) DFS of FGR level in early stage (stage I and II); (C) OS of FGR level in advanced stage (stage III and IV); (D) DFS of FGR level in advanced stage (stage III and IV). FGR, ferritin/globulin ratio; OS, overall survival; DFS, disease-free survival.

that are widely used in clinical laboratories. Previous studies demonstrated that over-expressed FERR has effect on multiple tumors, such as breast cancer, pancreatic cancer, Hodgkin's lymphoma and HCC. The serum FERR is a protein that stores iron and usually employed to assess iron homeostasis. The secretion source of serum FERR is still unclear, many studies demonstrated that hepatocytes, macrophages and microglia have the ability of FERR secretion $(13,14)$ and the most important is in macrophages $(15)$. The levels of serum ferritin are higher in patients with iron-overload disease and hemochromatosis (16), and the associations between coagulation system and cancer have been frequently studied. Several potential mechanisms have been postulated: (I) in tumor, the cancer cells with high proliferative potential is required to have higher demand for iron, which is essential for energy production and DNA synthesis. FERR is a very efficient iron delivery molecule with high iron storage capacity. (II) Immunosuppression , ferritin may act immunosuppressive effects on lymphocytes and myeloid cells through its modulation of iron availability $(17,18)$. One is that overexpression of iron decreases the numbers and activities of CD4 cells and increases the numbers and activities of suppressor T (CD8) cells resulting in increased CD8:CD4 ratios (19), suppresses the tumoricidal action of macrophages and monocytes (20). The other is high level FERR has been connected with elevated levels of anti-inflammatory cytokines, for example IL-10, whereas linked with the decreased of IL-2 (21). These proinflammatory cytokines are thought to support the cancer cells to evade the immune system in the tumor microenvironment. (III) The influence between FERR and high molecular weight kininogen (HK) could induced normal vessel formation to ensure sufficient vascularization of tumors $(22,23)$. (IV) Oxidative stress. The FERR released 

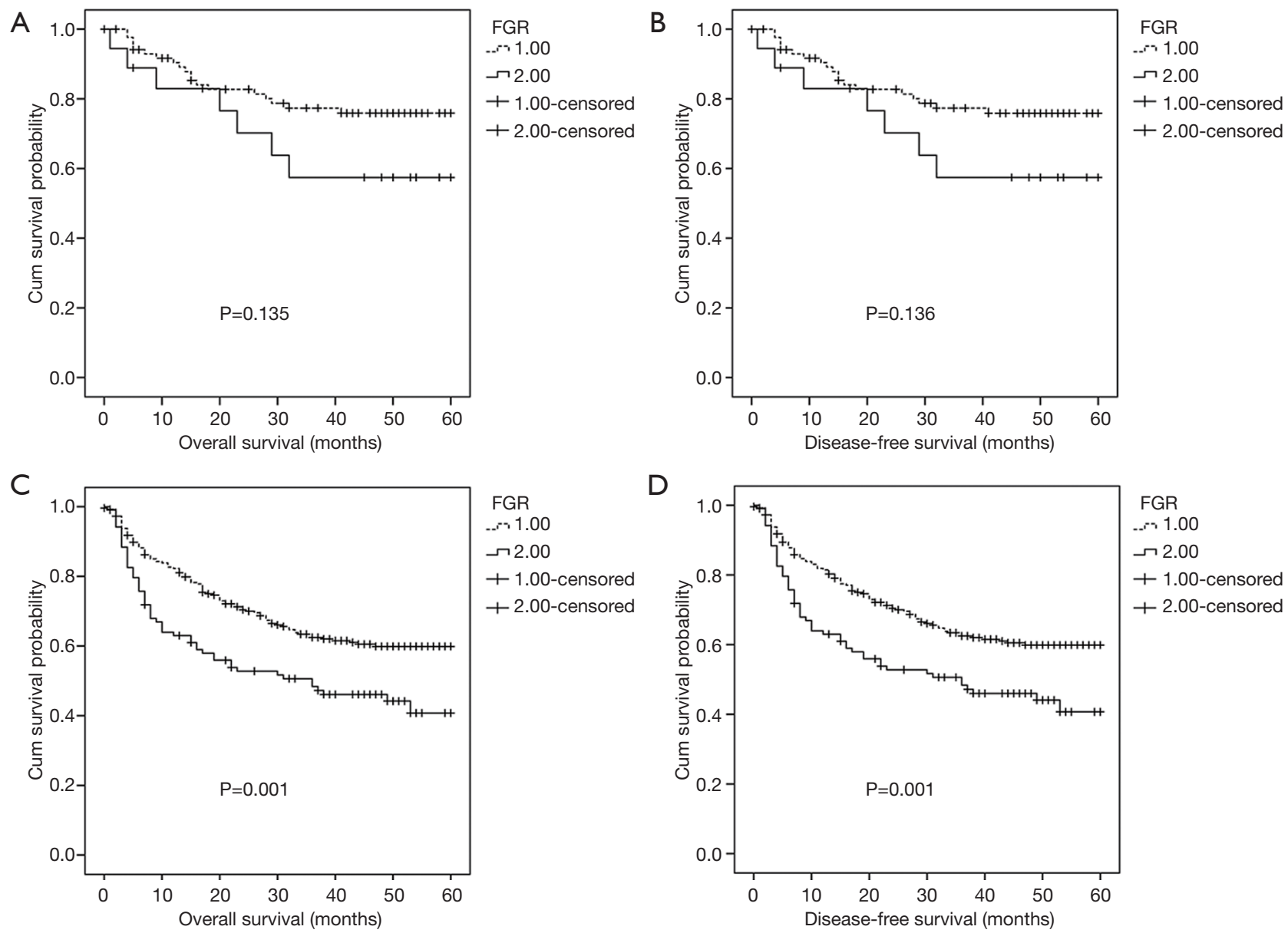

Figure 5 Prognosis significance of FGR level in different ALT group. (A) OS of FGR level in lower ALT group (<27.45 U/L); (B) DFS of FGR level in lower ALT group <27.45 U/L; (C) OS of FGR level in higher ALT group ( $\geq 27.45 \mathrm{U} / \mathrm{L}$ ); (D) DFS of FGR level in higher ALT group ( $\geq 27.45$ U/L). FGR, ferritin/globulin ratio; ALT, alanine aminotransferase; OS, overall survival; DFS, disease-free survival.

of ferric iron $\left(\mathrm{Fe}^{3+}\right)$, which reduced to ferrous iron $\left(\mathrm{Fe}^{2+}\right)$, and catalyzed the formation of the hydroxyl radical $\left({ }^{*} \mathrm{OH}\right)$ in the presence of superoxide and hydrogen peroxide $\left(\mathrm{H}_{2} \mathrm{O}_{2}\right)$. As a significant oxidizing agent, the hydroxyl radical can induce mutagenesis and DNA strand breaks, activate oncogenes, inhibit tumor suppressor gene, and promote lipid peroxidation (24). GLB was produced by immune organs, which has been used to reflect the immune state (25). It contained a lot of acute reactive proteins such as $\alpha_{1}$ antitrypsin, $\alpha_{2}$-macroglobulin, haptoglobin and so on. In the stimulation of inflammation, the level of serum GLB increased rapidly by several inflammatory indicator, it was also correlated with poor prognosis in several malignant tumors $(26,27)$.

Furthermore, the serum FERR level is influenced by acute and chronic inflammation, and is nonspecifically increased in a wide range of inflammatory conditions (28). Globulin is widely recognized as the biomarker of inflammation, so the level of FGR is better response to the impact of cancer on the body, which could weaken the influence of inflammation. Our study shows that FGR are more strongly associated with OS and DFS in HCC than FERR only. However, there were no significant correlations between FGR and AFP, the combination of AFP and FGR provide a new and effective method in HCC diagnosis and prognosis. Also, our results demonstrated that FGR related to AST and ALT, this may be because FERR, AST and ALT escaped from the impaired liver cancer in HCC (5).

In this study, the FGR level is higher in HCC patients than in normal controls. FGR could function as an independent prognostic factor of HCC, also both FERR and globulin are common and convenient monitoring tests 

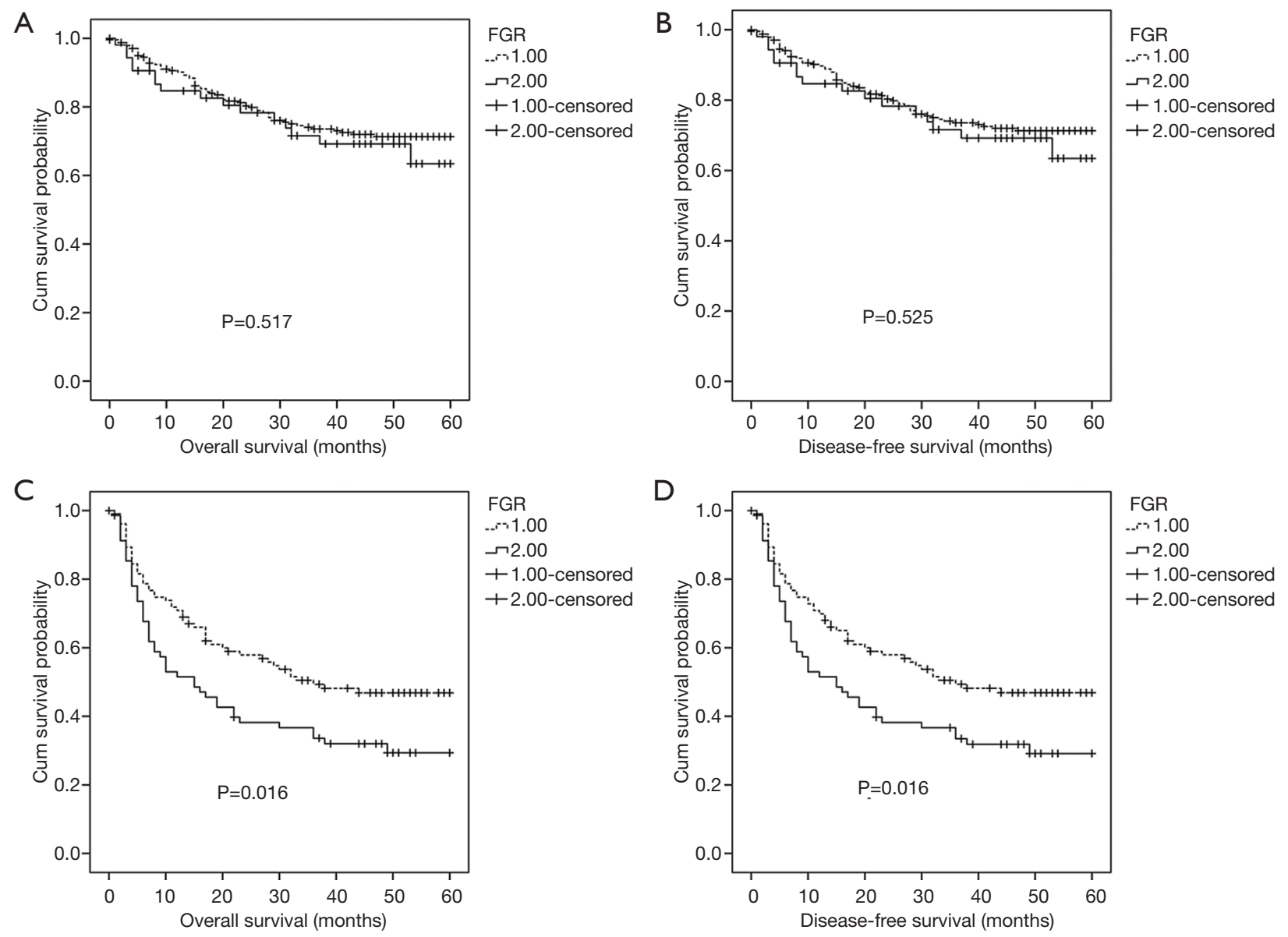

Figure 6 Prognosis significance of FGR level in different AST group. (A) OS of FGR level in lower AST group (<51.05 U/L); (B) DFS of FGR level in lower ALT group (<51.05 U/L); (C) OS of FGR level in higher ALT group ( $\geq 51.05$ U/L); (D) DFS of FGR level in higher ALT group ( $\geq 51.05 \mathrm{U} / \mathrm{L})$. FGR, ferritin/globulin ratio; AST, aspartate aminotransferase; OS, overall survival; DFS, disease-free survival; ALT, alanine aminotransferase.
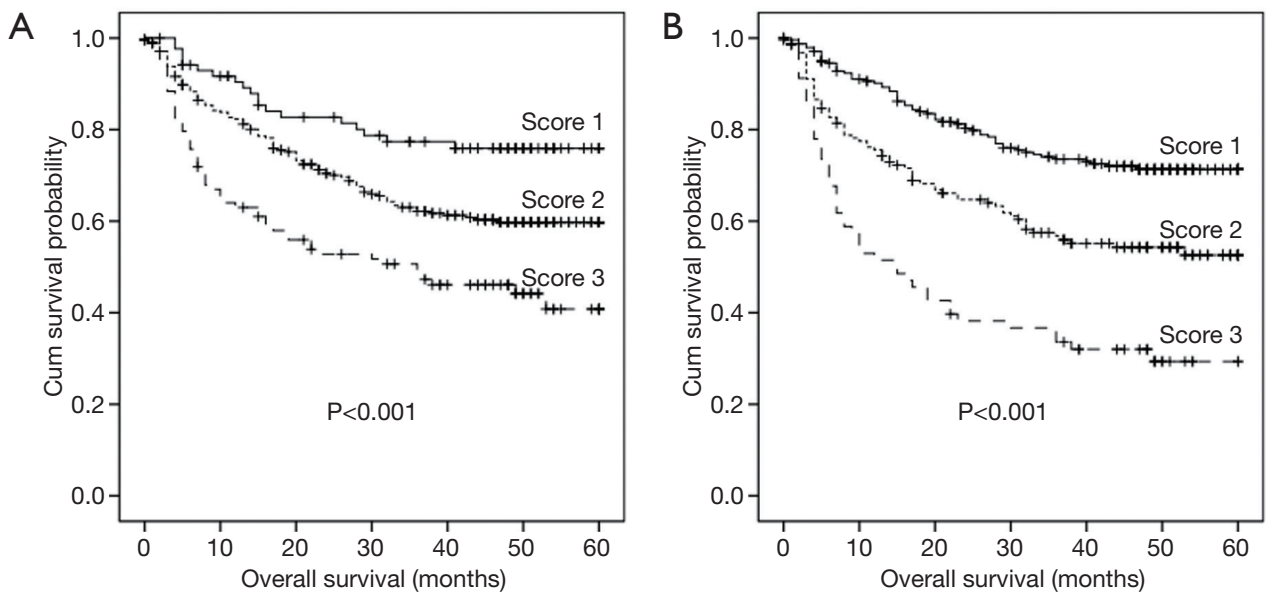

Figure 7 Prognosis value of combination of FGR and ALT/AST. (A) OS of FGR level combined with ALT; (B) OS of FGR level combined with AST. FGR, ferritin/globulin ratio; ALT, alanine aminotransferase; AST, aspartate aminotransferase; OS, overall survival. 
in routine preoperative examination. FGR can be employed as a prognostic tool for patients with HCC in combination with AFP.

\section{Acknowledgments}

Funding: This work was supported by grants from the Guangzhou science and technology planning project (201604016070).

\section{Footnote}

Reporting Checklist: The authors have completed the REMARK reporting checklist. Available at http://dx.doi. org/10.21037/tcr-20-966

Data Sharing Statement: Available at http://dx.doi. org/10.21037/tcr-20-966

Conflicts of Interest: All authors have completed the ICMJE uniform disclosure form (available at http://dx.doi. org/10.21037/tcr-20-966). The authors have no conflicts of interest to declare.

Ethical Statement: The authors are accountable for all aspects of the work in ensuring that questions related to the accuracy or integrity of any part of the work are appropriately investigated and resolved. The study was conducted in accordance with the Declaration of Helsinki (as revised in 2013). This study was approved by the Institute Research Ethics Committee of the Sun Yat-Sen University Cancer Center, Guangzhou, China (approval No. 2017 FXY-129). Each patient provided signed, informed consent.

Open Access Statement: This is an Open Access article distributed in accordance with the Creative Commons Attribution-NonCommercial-NoDerivs 4.0 International License (CC BY-NC-ND 4.0), which permits the noncommercial replication and distribution of the article with the strict proviso that no changes or edits are made and the original work is properly cited (including links to both the formal publication through the relevant DOI and the license). See: https://creativecommons.org/licenses/by-nc-nd/4.0/.

\section{References}

1. Velázquez RF, Rodriguez M, Navascues CA, et al. Prospective analysis of risk factors for hepatocellular carcinoma in patients with liver cirrhosis. Hepatology 2003;37:520-7.

2. Alisi A, Balsano C. Enhancing the efficacy of hepatocellular carcinoma chemotherapeutics with natural anticancer agents. Nutr Rev 2007;65:550-3.

3. Kabbach G, Assi HA, Bolotin G, et al. Hepatobiliary Tumors: Update on Diagnosis and Management. J Clin Transl Hepatol 2015;3:169-81.

4. Wang YG, Wang P, Wang B, et al. Diabetes mellitus and poorer prognosis in hepatocellular carcinoma: a systematic review and meta-analysis. PLoS One 2014;9:e95485.

5. Kew MC, Torrance JD, Derman D, et al. Serum and tumour ferritins in primary liver cancer. Gut 1978;19:294-9.

6. Weinstein RE, Bond BH, Silberberg BK. Tissue ferritin concentration in carcinoma of the breast. Cancer 1982;50:2406-9.

7. Lambrecht RW, Sterling RK, Naishadham D, et al. Iron levels in hepatocytes and portal tract cells predict progression and outcomes of patients with advanced chronic hepatitis C. Gastroenterology 2011;140:1490-500.e3.

8. Ganz T, Nemeth E. Iron sequestration and anemia of inflammation. Semin Hematol 2009;46:387-93.

9. Li Q, Meng X, Liang L, et al. High preoperative serum globulin in rectal cancer treated with neoadjunctive chemoradiation therapy is a risk factor for poor outcome. Am J Cancer Res 2015;5:2856-64.

10. Tsurusaki M, Murakami T. Surgical and Locoregional Therapy of HCC: TACE. Liver Cancer 2015;4:165-75.

11. Laurent C, Blanc JF, Nobili S, et al. Prognostic factors and longterm survival after hepatic resection for hepatocellular carcinoma originating from noncirrhotic liver. J Am Coll Surg 2005;201:656-62.

12. Song P, Feng X, Zhang K, et al. Perspectives on using desgamma-carboxyprothrombin (DCP) as a serum biomarker: facilitating early detection of hepatocellular carcinoma in China. Hepatobiliary Surg Nutr 2013;2:227-31.

13. De Domenico I, Vaughn MB, Paradkar PN, et al. Decoupling ferritin synthesis from free cytosolic iron results in ferritin secretion. Cell Metab 2011;13:57-67.

14. Tran TN, Eubanks SK, Schaffer KJ, et al. Secretion of ferritin by rat hepatoma cells and its regulation by inflammatory cytokines and iron. Blood 1997;90:4979-86.

15. Zhang Z, Zhang F, An P, et al. Ferroportin1 deficiency in mouse macrophages impairs iron homeostasis and inflammatory responses. Blood 2011;118:1912-22.

16. Rienhoff HY Jr. Iron-overload-related disease in HFE hereditary hemochromatosis. N Engl J Med 2008;358:2294; author reply 2294-5. 
17. Fargion S, Fracanzani AL, Brando B, et al. Specific binding sites for H-ferritin on human lymphocytes: modulation during cellular proliferation and potential implication in cell growth control. Blood 1991;78:1056-61.

18. Matzner Y, Hershko C, Polliack A, et al. Suppressive effect of ferritin on in vitro lymphocyte function. Br J Haematol 1979;42:345-53.

19. Walker EM Jr, Walker SM. Effects of iron overload on the immune system. Ann Clin Lab Sci 2000;30:354-65.

20. Weinberg JB, Hibbs JJ. Endocytosis of red blood cells or haemoglobin by activated macrophages inhibits their tumoricidal effect. Nature 1977;269:245-7.

21. Gray CP, Franco AV, Arosio P, et al. Immunosuppressive effects of melanoma-derived heavy-chain ferritin are dependent on stimulation of IL-10 production. Int J Cancer 2001;92:843-50.

22. Tesfay L, Huhn AJ, Hatcher H, et al. Ferritin blocks inhibitory effects of two-chain high molecular weight kininogen $(\mathrm{HKa})$ on adhesion and survival signaling in endothelial cells. PLoS One 2012;7:e40030.

23. Coffman LG, Parsonage D, D'agostino RJ, et al.

Cite this article as: Liu W, Chen Q, Mao M, Han R, Liu Y, Wang X. Novel prognostic scores based on serum ferritin/ globulin ratio in patients with hepatocellular carcinoma. Transl Cancer Res 2020;9(10):5925-5939. doi: 10.21037/tcr-20-966
Regulatory effects of ferritin on angiogenesis. Proc Natl Acad Sci U S A 2009;106:570-5.

24. Kabat GC, Rohan TE. Does excess iron play a role in breast carcinogenesis? An unresolved hypothesis. Cancer Causes Control 2007;18:1047-53.

25. Ballow M. Mechanisms of action of intravenous immune serum globulin in autoimmune and inflammatory diseases. J Allergy Clin Immunol 1997;100:151-7.

26. Adly L, Hill D, Sherman ME, et al. Serum concentrations of estrogens, sex hormone-binding globulin, and androgens and risk of breast cancer in postmenopausal women. Int J Cancer 2006;119:2402-7.

27. Sawada N, Iwasaki M, Inoue M, et al. Plasma testosterone and sex hormone-binding globulin concentrations and the risk of prostate cancer among Japanese men: a nested casecontrol study. Cancer Sci 2010;101:2652-7.

28. Kalantar-Zadeh K, Kalantar-Zadeh K, Lee GH. The fascinating but deceptive ferritin: to measure it or not to measure it in chronic kidney disease? Clin J Am Soc Nephrol 2006;1 Suppl 1:S9-18. 